\title{
ANATOMIA DO LENHO DE MIMOSA MICROPTERIS BENTH. ${ }^{1}$
}

\author{
ANE CAROLINE DA SILVA PEREIRA² ANELISE MARTA SIEGLOCH ${ }^{3}$ \\ JOSÉ NEWTON CARDOSO MARCHIORI ${ }^{4}$
}

\section{RESUMO}

A anatomia da madeira de Mimosa micropteris Benth. é presentemente descrita com base em material procedente de Sengés, Paraná, e de acordo com IAWA (1989). A estrutura da madeira apresenta: placas de perfuração simples; elementos vasculares curtos; pontoações intervasculares alternas e ornamentadas; parênquima paratraqueal; raios heterogêneos; fibras libriformes; e estrutura não estratificada.

Palavras-chave: Anatomia da madeira, Mimosa micropteris, Fabaceae.

\section{ABSTRACT}

[Wood anatomy of Mimosa micropteris Benth.].

The wood of Mimosa micropteris is anatomically described, based on material from Sengés, Paraná state (Brazil), and according to IAWA (1989). The wood structure presents: simple perforation plates; short vascular elements; alternate vestured pits; paratracheal parenchyma; heterogeneous rays; libriform fibers; and non storied structure.

Key words: Wood anatomy, Mimosa micropteris, Fabaceae.

\section{INTRODUÇÃO}

A família Fabaceae inclui cerca de 650 gêneros e aproximadamente 18.000 espécies, distribuídas em todas as partes do mundo (Souza \& Lorenzi, 2008), com exceção das regiões árticas e antárticas. São muito abundantes em países tropicais como o Brasil, onde se destacam na composição de matas nativas e reúnem centenas de espécies produtoras de madeiras valiosas (Marchiori, 2007).

Motivo de controvérsias, a taxonomia das Fabaceae inclui, tradicionalmente, três subfamílias: Papilionoideae ou Faboideae, Caesalpinioideae e Mimosoideae (Souza \& Lorenzi, 2008). A subfamília Mimosoideae comprende de 50 a 60 gêneros (Marchiori,

1 Recebido para publicação em 14/02/2013 e aceito para publicação em 30/04/2013.

2 Acadêmica do curso de Engenheira Florestal. Bolsista de Iniciação Científica (PIBIC - CNPq). Universidade Federal de Santa Maria. Santa Maria, RS, Brasil. aneh.caroline@hotmail.com

3 Mestranda do Programa de Pós-Graduação em Engenharia Florestal. Bolsista - CAPES. Universidade Federal de Santa Maria. Santa Maria, RS, Brasil.

${ }^{4}$ Engenheiro Florestal, Dr. Bolsista de Produtividade em Pesquisa $(\mathrm{CNPq}-$ Brasil). Professor Titular do Departamento de Ciências Florestais, Universidade Federal de Santa Maria. Santa Maria, RS, Brasil.
2007), e o gênero Mimosa, cerca de 500 espécies (Judd et al., 2009).

Conhecida pelo nome comum de juquiri (Burkart, 1979), Mimosa micropteris Benth. foi descrita em 1841, no Journal of Botany, de Hooker (n. 4, p. 384), com base em tipo coletado por Sellow (n. 5.150), conservado em Kew.

Trata-se de arbusto ou arvoreta de 1-4 m de altura, inerme, com folhas de 8-23-jugas, flores em capítulos globosos reunidos em racemos, e legumes comprimidos de 12-18 x 5-6,5 mm, com valvas deiscentes, papiráceas, que não se desarticulam em segmentos subquadrados (Burkart, 1979).

De acordo com Barneby (1991), a espécie inclui, atualmente, três táxones anteriormente reconhecidos como distintos, por Burkart (Mimosa micropteris sens. str., $M$. regnelii sensu Burkart (non Benth.), e M. regnelii var. pungens Burkart). Trata-se de espécie extratropical, com larga distribuição nos "Campos Gerais" do segundo planalto paranaense, desde a divisa de São Paulo (curso superior do rio Itararé), até o centro-leste de Santa Catarina (Barneby, 1991).

Sob o ponto de vista taxonômico, Mimosa micropteris pertence à Série Myriophyllae e Seção Mimosa, juntamente com Mimosa 
balduinii, espécie endêmica da borda dos Aparados da Serra (RS), Mimosa myriophylla, $M$. glazioui e $M$. altoparanensis, todas nativas em terras altas do Planalto Sul-Brasileiro.

A anatomia da madeira de Mimosa micropteris é desconhecida, não constando na literatura igualmente qualquer referência anatômica sobre espécies da Série Myriophyllae.

Em estudo do lenho de 11 Mimosas da Argentina, Cozzo (1951) definiu o gênero como estruturalmente heterogêneo, devido à ampla variação observada. Nenhuma das espécies estudadas, todavia, pertence à Série Myriophyllae.

Com mais de 80 espécies nativas no sul do Brasil, são relativamente escassas as espécies de Mimosa para as quais foram descritos caracteres microscópicos do lenho, salientandose, neste aspecto: Mimosa daleoides (Marchiori, 1882); M. cruenta (Marchiori, 1985); $M$. bimucronata (Marchiori, 1980, 1993); M. eriocarpa (Carnieletto \& Marchiori, 1993): $M$. sparsa (Maccari \& Marchiori, 1994): M. scabrella (1980, 1995); M. berroi (Marchiori, 1996a); M. uruguensis (Marchiori (1996b); M. incana (Marchiori, 1996c); M. trachycarpa (Marchiori \& Muniz, 1997a); e M. pilulifera (Marchiori \& Muniz, 1997b).

\section{MATERIAL E MÉTODOS}

O material em estudo consiste de uma amostra de madeira e respectivo material botânico, conservados no Herbário do Departamento de Ciências Florestais da Universidade Federal de Santa Maria (HDCF) sob o número 3175. Na etiqueta de herbário, constam os seguintes dados: Cerrado de Sengés, PR, Marchiori, J. N. C., 13-05-1987.

Para a confecção de lâminas histológicas foram extraídos três corpos de prova $(1 \times 2 \times 3 \mathrm{~cm})$ da parte mais externa do lenho, próxima ao câmbio, orientados para obtenção de cortes nos planos transversal, longitudinal radial e longitudinal tangencial. Outro bloquinho foi também retirado, com vistas à maceração.
O preparo de lâminas histológicas seguiu a metodologia descrita em Burger \& Richter (1991). A maceração foi realizada pelo método de Jeffrey (Freund, 1970). Os cortes anatômicos foram tingidos com acridina-vermelha, crisoidina e azul-de-astra (Dujardin, 1964); o macerado, apenas com safranina (1\%). A montagem de lâminas permanentes foi feita com Entellan.

A descrição baseou-se nas recomendações do IAWA Committee (Wheeler et al., 1989). No caso da percentagem dos tecidos, foram realizadas 600 determinações ao acaso, com auxílio de contador de laboratório, conforme proposto por Marchiori (1980). A abundância de poros foi obtida a partir de um quadrado de área conhecida, superposto a fotomicrografias de seções transversais da madeira.

As medições foram realizadas em microscópio Carl Zeiss, no Laboratório de Anatomia da Madeira da Universidade Federal de Santa Maria. Nas características quantitativas, os números entre parênteses equivalem aos valores mínimos e máximos observados; o valor que acompanha a média é o desvio padrão. As fotomicrografias foram tomadas em microscópio Olympus CX40, equipado com câmera digital Olympus Camedia c3000, no Laboratório de Anatomia da Madeira da Universidade Federal do Paraná.

\section{DESCRIÇÃO ANATÔMICA}

Madeira de porosidade difusa. Anéis de crescimento indistintos (Figura 1A,B).

Vasos: numerosos $(48 \pm 18,4(25-75)$ po$\operatorname{ros} / \mathrm{mm}^{2}$ ), ocupando $18,5 \pm 3,3 \%$ do volume da madeira. Poros solitários em sua maioria, em múltiplos radiais $2-4$, menos comumente racemiformes, arredondados ( $80 \pm 13,6(51-$ $100) \mu \mathrm{m})$ e de paredes espessas $(4,6 \pm 0,9(3,1$ $-6,2) \mu \mathrm{m}$ ) (Figura 1A,B). Elementos vasculares curtos $(225 \pm 45(140-350) \mu \mathrm{m})$, com placas de perfuração simples, transversais ao vaso, e apêndices curtos, geralmente em uma extremidade. Pontoações intervasculares pequenas $(5,2$ 

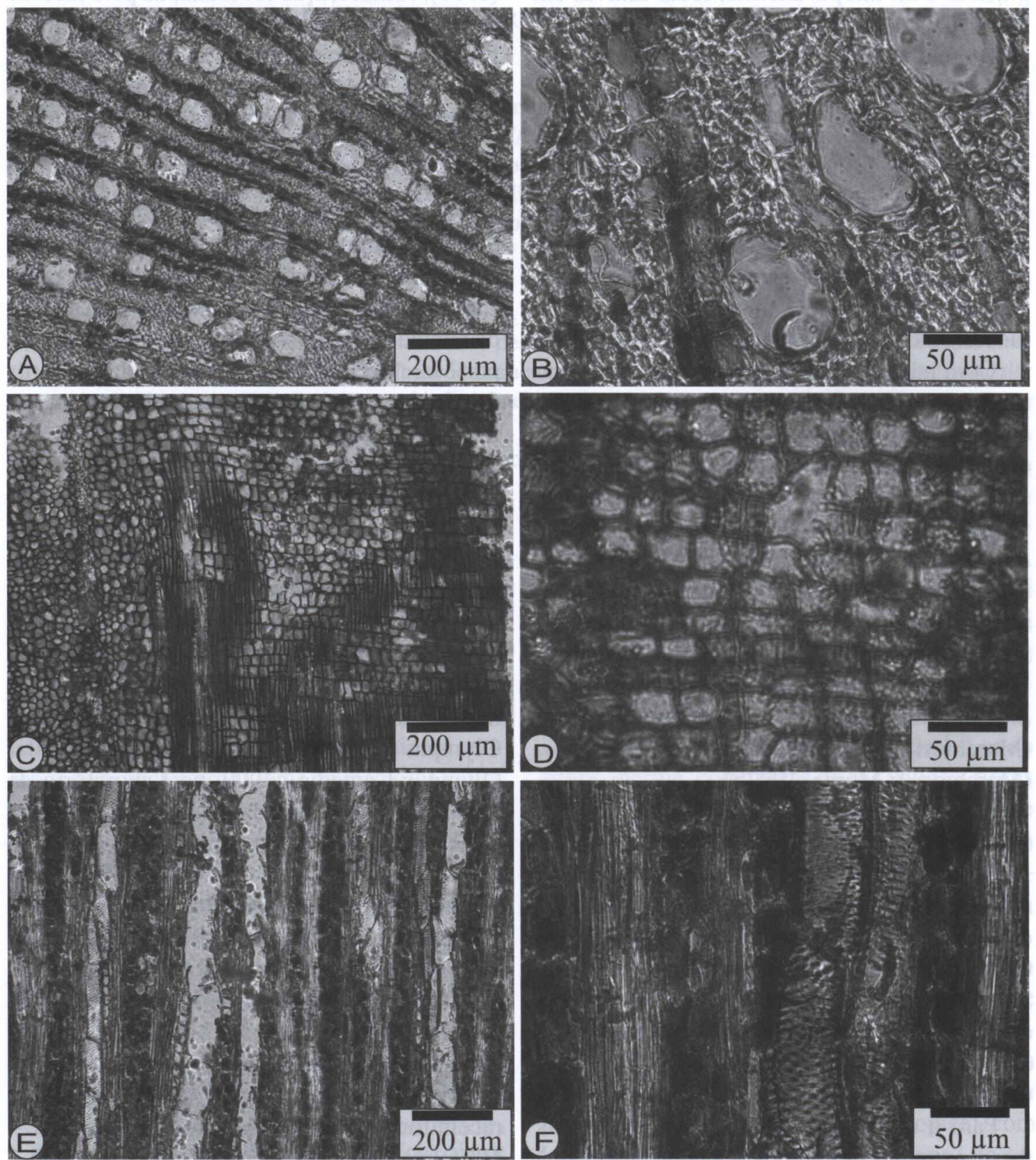

FIGURA 1 - Fotomicrografias do lenho de Mimosa micropteris. A - Poros solitários e raios com conteúdo (secção transversal). B - Mesma secção, destacando poros solitários. C - Aspecto geral de raio heterogêneo, com células quadradas, eretas e procumbentes (secção longitudinal radial). D - Mesma secção, em maior aumento. E - Raios uni e multisseriados e elementos de vaso (secção longitudinal tangencial). F - Elementos de vaso, fibras e parte de raios multisseriados (secção longitudinal tangencial). 
$\pm 0,8(4-6,2) \mu \mathrm{m})$, alternas, circulares, por vezes coalescentes, com abertura em fenda inclusa, ornamentada (Figura 1F). Espessamentos espiralados, ausentes. Conteúdo em vasos, escasso.

Parênquima axial: representando 7,5 $\pm 2,1$ $\%$ do volume da madeira; em arranjo paratraqueal-escasso (Figura 1A,B). Células fusiformes de $170 \pm 30(107-220) \mu \mathrm{m}$. Séries parenquimáticas de $233 \pm 62,6(115-375) \mu \mathrm{m}$ de altura, com 2-4 células (Figura 1F). Cristais romboédricos, abundantes, em 8-14 câmaras por série.

Raios: numerosos $(11 \pm 1,7(9-14)$ raios/ $\mathrm{mm}$ ), com 1-5 células de largura, ocupando 33,3 $\pm 5,7 \%$ do volume da madeira (Figura 1E,F). Os multisseriados, de $1125 \pm 539(434-2460)$ $\mu \mathrm{m}$ e $31-128$ células de altura; heterocelulares, reúnem células quadradas e eretas, por vezes procumbentes (Figura 1C,E,F). Os unisseriados, de $372 \pm 155,7(168-841) \mu \mathrm{m}$ e $6-34$ células de altura. Raios fusionados, escassos. Raios agregados, raros. Conteúdo, abundante. Células envolventes, células radiais de paredes disjuntas e células perfuradas, ausentes.

Fibras: com pequenas pontoações simples; curtas $(532 \pm 92,5(300-811) \mu \mathrm{m}), \operatorname{com} 9 \pm 2,1$ $(6-14) \mu \mathrm{m}$ de largura, e paredes finas a espessas $3,2 \pm 1,4(2,5-5) \mu \mathrm{m}$, ocupando $40,7 \pm 4,5$ $\%$ do volume da madeira (Figura 1B). Fibras gelatinosas, espessamentos espiralados, fibras septadas e traqueídeos, ausentes.

Outros caracteres: variantes cambiais, tubos laticíferos e taniníferos, canais intercelulares, células oleíferas, células mucilaginosas, estratificação, e máculas medulares, ausentes. Cristais, presentes.

\section{ANÁLISE DA ESTRUTURA ANATÔMICA}

A estrutura anatômica de Mimosa micropteris indica alto grau de especialização e corresponde, em seus caracteres mais relevantes, ao descrito por Record \& Hess (1949) e Metcalfe \& Chalk (1972) para a família Leguminosae: placas de perfuração simples; elementos vasculares curtos; pontoações intervasculares alternas, ornamentadas; parênquima paratraqueal; e fibras libriformes.

A presença de tecido radial fracamente heterogêneo na espécie em estudo foge ao padrão característico das Mimosoídeas. BarettaKuipers (1982) ressalta que a homogeneidade dos raios é a característica mais notável da subfamília e uma importante tendência evolutiva. Não obstante, raios heterogêneos são mencionados para diversas espécies do gênero, incluindo Mimosa scabrella (Marchiori, 1980, 1995), M. daleoides (Marchiori, 1982), M. eriocarpa (Carnieletto \& Marchiori, 1993), M. incana (Marchiori, 1996) e M. pilulifera (Marchiori, 1997).

Carnieletto \& Marchiori, (1993) relacionaram a presença de raios com células quadradas e eretas a estágios iniciais da ontogenia, em plantas jovens (pedomorfia). No material estudado, a presença de medula nos cortes anatômicos corrobora esta interpretação.

Baretta-Kuipers (1980; 1981), por sua vez, ressalta a ausência de estratificação como caráter indicativo da sub-família Mimosoideae.

\section{REFERÊNCIAS BIBLIOGRÁFICAS}

BARETTA-KUIPERS,T. The wood structure of leguminous tribes: their classification by ray and parenchyma features. For. Prod. Abstr., v.3, n.8, p.1-784, 1980.

BARETTA-KUIPERS,T. Wood anatomy of Leguminosae: its relevance to Taxonomy. In: POLHILL, R. M. \& RAVEN, P.H. Advances in Legume Systematics. 1981. p.677-715.

BARNEBY, R.C. Sensitivae Censitae. A description of the genus Mimosa Linnaeus (Mimosaceae) in the New World. Memoirs of the New York Botanical Garden, v. 65, p. 1-835, 1991.

BURGER, L.M.; RICHTER, H.G. Anatomia da Madeira. São Paulo: Ed. Nobel, 1991. 154 p.

BURKART, A. Leguminosas Mimosoideas. In: REITZ, P.R. Flora Ilustrada Catarinense. Itajaí: Herbário Barbosa Rodrigues, 1979. 299 p.

CARNIELETTO, C.; MARCHIORI, J.N.C. Anatomia da madeira de Mimosa eriocarpa Benth. $\mathrm{Ci}$ ência Florestal, Santa Maria, v. 3, n. 1, p. 107120, 1993. 
COZZO, D. Anatomia del leño secundario de las Leguminosas Mimosoideas y Cesalpinioideas argentinas silvestres y cultivadas. Revista del Inst. Nac. de Invest. de las Ciencias Naturales, Ciencias Botánicas, Buenos Aires, v. 2, n. 2, p. 63-146, 1951.

DUJARDIN, E.P. Eine neue Holz-Zellulosenfaerbung. Mikrokosmos, n. 53, p. 94, 1964.

FREUND, H. Handbuch der Mikroskopie in der Technik. Frankfurt: Umsham Verlag, 1970.

JUDD, W. S.; CAMPBELL, C. S.; KELLOGG, E. A.; STEVENS, P. F.; DONOGHUE, M. J. Sistemática vegetal: um enfoque filogenético. Porto Alegre: Artmed, 2009. p. 371-377.

MACCARI, A.; MARCHIORI, J.N.C. Estudo anatômico do xilema secundário de Mimosa sparsa Benth. Ciência Florestal, Santa Maria, v. 4, p. 145-155, 1994.

MARCHIORI, J.N.C. A estrutura do xilema secundário de Mimosa daleoides Benth. (Leguminosae Mimosoideae). Ciência e Natura, Santa Maria, v. 4, p. 107-113, 1982.

MARCHIORI, J.N.C. Anatomia da madeira de $\mathrm{Mi}$ mosa cruenta Benth. (Leguminosae Mimosoideae). Ciência e Natura, Santa Maria, v. 7, p. 73-81, 1985.

MARCHIORI, J.N.C. Anatomia da madeira e casca do maricá, Mimosa bimucronata (DC.) O. Kuntze. Ciência Florestal, Santa Maria, v. 3, n. 1, p. 85-106, 1993.

MARCHIORI, J.N.C. Anatomia do xilema secundário de Mimosa berroi Burk. Ciência e Natura, Santa Maria, v. 18, p. 117-129, 1996a.
MARCHIORI, J.N.C. Anatomia do xilema secundário de Mimosa incana (Spreng.) Benth. Ciência Florestal, Santa Maria, v. 6, n. 1, p. 53-63, 1996 c.

MARCHIORI, J.N.C. Anatomia do xilema secundário de Mimosa uruguensis Hook. et Arn. Ciência e Natura, Santa Maria, v. 18, p. 103-115, 1996 b.

MARCHIORI, J.N.C. Estudo anatômico do xilema secundário e da casca de algumas espécies dos gêneros Acacia e Mimosa, nativas no Estado do Rio Grande do Sul. Curitiba: UFPR, 1980. 185 f. Dissertação (Mestrado em Engenharia Florestal - Universidade Federal do Paraná).

MARCHIORI, J.N.C.; MUÑIZ, G.I.B. de. Anatomia do xilema secundário de Mimosa pilulifera Benth. Ciência Florestal, Santa Maria, v. 6, n. 1, p. 65-75, $1997 \mathrm{~b}$.

MARCHIORI, J.N.C.; MUÑIZ, G.I.B. de. Estudo anatômico do xilema secundário de Mimosa trachycarpa Benth. Ciência Rural, Santa Maria, v. 27, n. 2, p. 223-228, 1997a.

METCALFE, C.R.; CHALK, L. Anatomy of the Dicotyledons. Oxford: Clarendon Press, 1972. p. 476-487.

RECORD, S.J.; HESS, R.W. Timbers of the New World. New Haven: Yale University Press, 1949. 640 p.

SOUZA, V. C.; LORENZI, H. Botânica Sistemáti$c a$ : guia ilustrado para identificação das famílias de Angiospermas da flora brasileira, baseado em APG II. Nova Odessa: Instituto Plantarum, 2008. p. 381-422.

WHEELER, E.A.; BAAS, P.; GASSON, P.E. IAWA list of microscopic features for hardwood identification. IAWA Bulletin, v.10, n. 3, 1989. p. 218-359. 